\title{
Toxicity of Cryoprotectants to Gametophytic Thalli of Red Algae Porphyra yezoensis
}

\author{
Youn Hee Choi ${ }^{1}$ and Taek Jeong $\mathrm{Nam}^{2 *}$ \\ ${ }^{1}$ Fisheries Science Research Center, Pukyong National University, Busan 619-911, Korea \\ ${ }^{2}$ Department of Food Science and Nutrition, Pukyong National University, Busan 608-737, Korea
}

\begin{abstract}
We assessed the toxicity of cryoprotectant agents (CPAs) to gametophytic thalli of red alga Porphyra yezoensis at room temperature. The CPAs used were: dimethyl sulfoxide (DMSO), ethylene glycol (EG), glycerol (GC), 1,2-butanediol (1,2-BD), 1,3-butanediol (1,3-BD), 2,3-butanediol (2,3-BD), 1,3-propanediol (1,3-PD) and propylene glycol (PG). CPA concentrations of 10, 15, $20,25,30,35,40,45$, and $50 \%$ were employed with 30 or $60 \mathrm{~s}$ immersion. The toxicity of the eight CPAs to gametophytic thalli of $P$. yezoensis was in the order: $1,3-\mathrm{BD}<\mathrm{DMSO} \approx 2,3-\mathrm{BD} \approx \mathrm{PG} \approx \mathrm{EG}<\mathrm{GC}<1,3-\mathrm{PD} \approx 1,2-\mathrm{BD}$. All thalli were more sensitive to high CPA concentrations, and most ( $>75 \%$ ) thalli survived exposure to $10-25 \%$ CPA for $60 \mathrm{~s}$. These data will facilitate selection of the optimal cryoprotectant concentration for cryopreservation of $P$. yezoensis thalli.
\end{abstract}

Key words: Red algae, Porphyra yezoensis, Cryoprotectant agents, Toxicity

\section{Introduction}

Cryoprotectant agents (CPAs) are chosen because of their useful non-crystallization property of hydrate formation at low temperatures in aqueous solution (Wowk, 2010). However, the toxicity of which is a fundamental limiting factor for the successful cryopreservation of living cells (Fahy, 2010). So far, most reports of CPA toxicities were focused on finfishes such as flounder embryos/sperm (Zhang et al., 2005), zebrafish (Zhang and Rawson, 1993; Liu et al., 2001), medaka (Yang et al., 2010), and so on. However, little research on the toxicity of CPAs to macroalgae has been conducted.

In order to determine the tolerance of gametophytic thalli of Porphyra yezoensis to their toxicity without freezing, we used eight CPAs (sulfoxide, diol and tiol groups) - dimethyl sulfoxide (DMSO), ethylene glycol (ethane-1,2-diol, EG), glycerol (propan-1,2,3-tiol, GC), 1,2-butanediol (1,2-BD), 1,3-butanediol (1,3-BD), 2,3-butanediol (2,3-BD), propylene glycol (1,2-propanediol, PG) and 1,3-propanediol (1,3-PD).

\section{Materials and Methods}

\section{Algal strains and culture conditions}

Gametophytic thalli of $P$. yezoensis were obtained from TU-1 (Kuwano et al., 1996). Thalli were cultured in a 1 L flask containing Provasoli's enriched seawater medium (Provasoli, 1968 ) and pieces of synthetic fiber (3-5 cm in length, $0.25 \mathrm{~mm}$ in diameter) at $15^{\circ} \mathrm{C}$ and irradiated at $60 \mu \mathrm{mol}$ photons $\mathrm{m}^{-2} \mathrm{~s}^{-1}$ with a 10:14 h light:dark photoperiod for two weeks. Monospores were released from thalli attached to the synthetic fibers and grew into young thalli of identical genotype.

\section{CPA toxicity test}

Nine concentrations $(10,15,20,25,30,35,40,45$ and $50 \%)$ of each of the eight CPAs (DMSO, EG, GC, 1,2-BD, 1,3-BD,
Open Access http://dx.doi.org/10.5657/FAS.2012.0077

This is an Open Access article distributed under the terms of the Creative Commons Attribution Non-Commercial License (http:// creativecommons. org/licenses/by-nc/3.0/) which permits unrestricted non-commercial use distribution, and reproduction in any medium, provided the original work is properly cited.
Received 8 November 2011; $\quad$ Revised 2 February 2012; Accepted 9 February 2012

*Corresponding Author

E-mail: namtj@pknu.ac.kr 
2,3-BD, PG and 1,3-PD) were tested. All chemicals were reagent-grade, and cryoprotectant solutions were prepared in 0.2 $\mu \mathrm{m}$-filtered seawater. Synthetic fibers with attached thalli were cut to $0.5-1 \mathrm{~cm}$ lengths and transferred to CPA solutions for 30 or $60 \mathrm{~s}$. Thalli were then stained with $0.1 \%$ erythrosine (in seawater) for 20 min and washed with fresh seawater.

Thalli viability was examined under a light microscope (BX-50; Olympus, Tokyo, Japan). The survival rates were ex- pressed as the ratio of the number of living thalli to the total number observed.

\section{Results and Discussion}

Fig. 1 shows survival of the gametophytic thalli of $P$. yezoensis according to CPA toxicity. The ability of thalli to

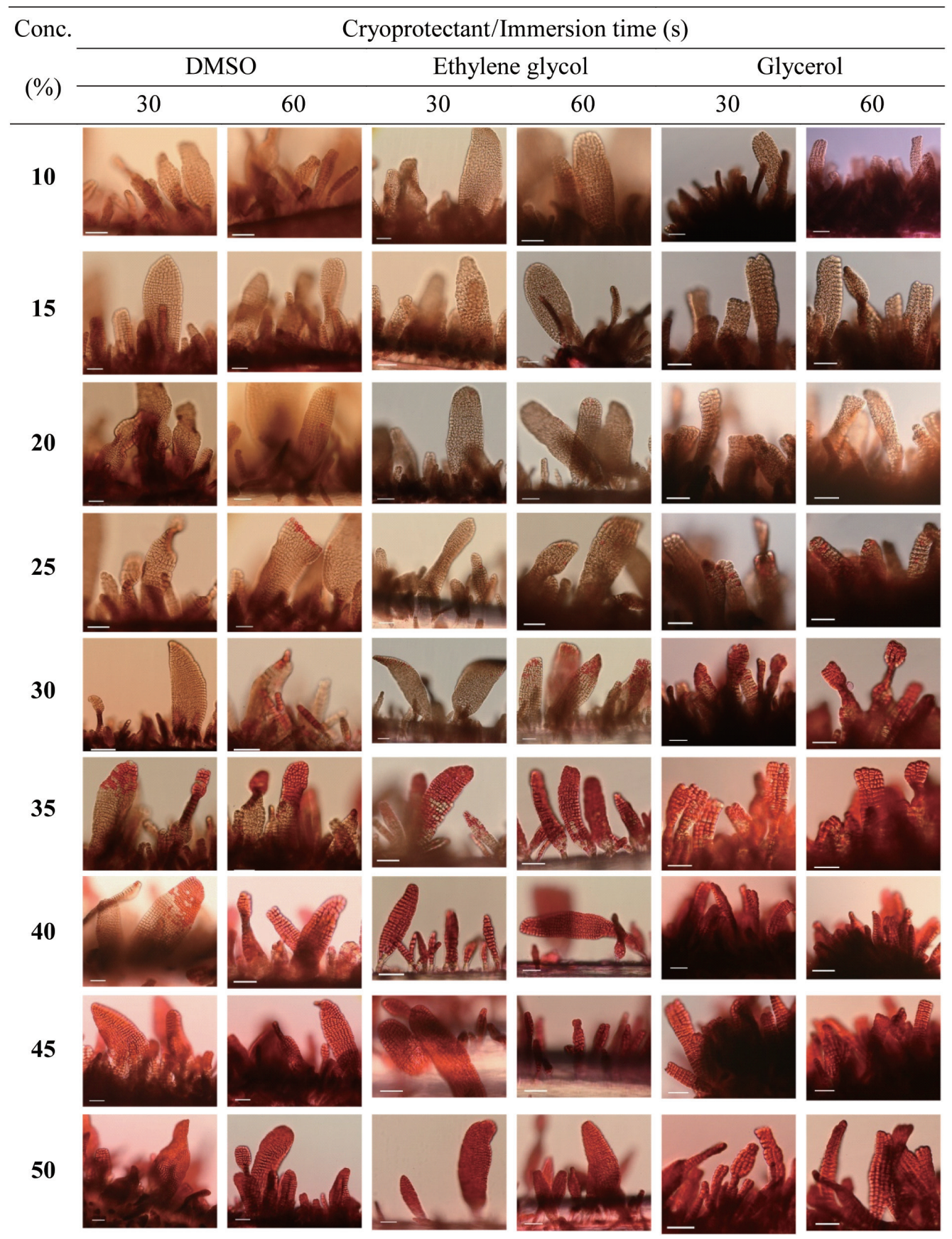

Fig. 1. Toxicity of different concentrations of cryoprotectants to gametophytic thalli of Porphyra yezoensis illustrated as photograph. DMSO, dimethyl sulfoxide. Scale bars $=50 \mu \mathrm{m}$. 
survive exposure to CPAs differed, and their tolerance of CPA toxicity tended to decrease with increasing CPA concentration. The thalli showed the highest tolerance to $1,3-\mathrm{BD}$, and the lowest to 1,2-BD. Glycerol and 1,3-PD were lethal to thalli at $40 \%$, and no thalli survived exposure to $35 \% 1,2-\mathrm{BD}$. However, thalli maintained viability ( $>70 \%$ ) when exposed to $50 \%$ 1,3-BD for $30 \mathrm{~s}$. Therefore, 1,3-BD exhibited the lowest toxicity, followed by DMSO, 2,3-BD, PG and 1,2-BD.
In aqueous solutions, 2,3-BD exhibits glass formation superior to that of other CPA, including 1,2-PD and 1,3-PD (Baudot et al., 1996). Shaw et al. (1995) reported that propanediol forms a stable vitreous state on cooling to a greater degree than other CPA in human and mouse embryos. We found that gametophytic thalli of $P$. yezoensis were highly tolerant of 1,3$\mathrm{BD}$, even though the determinations were conducted at room temperature.

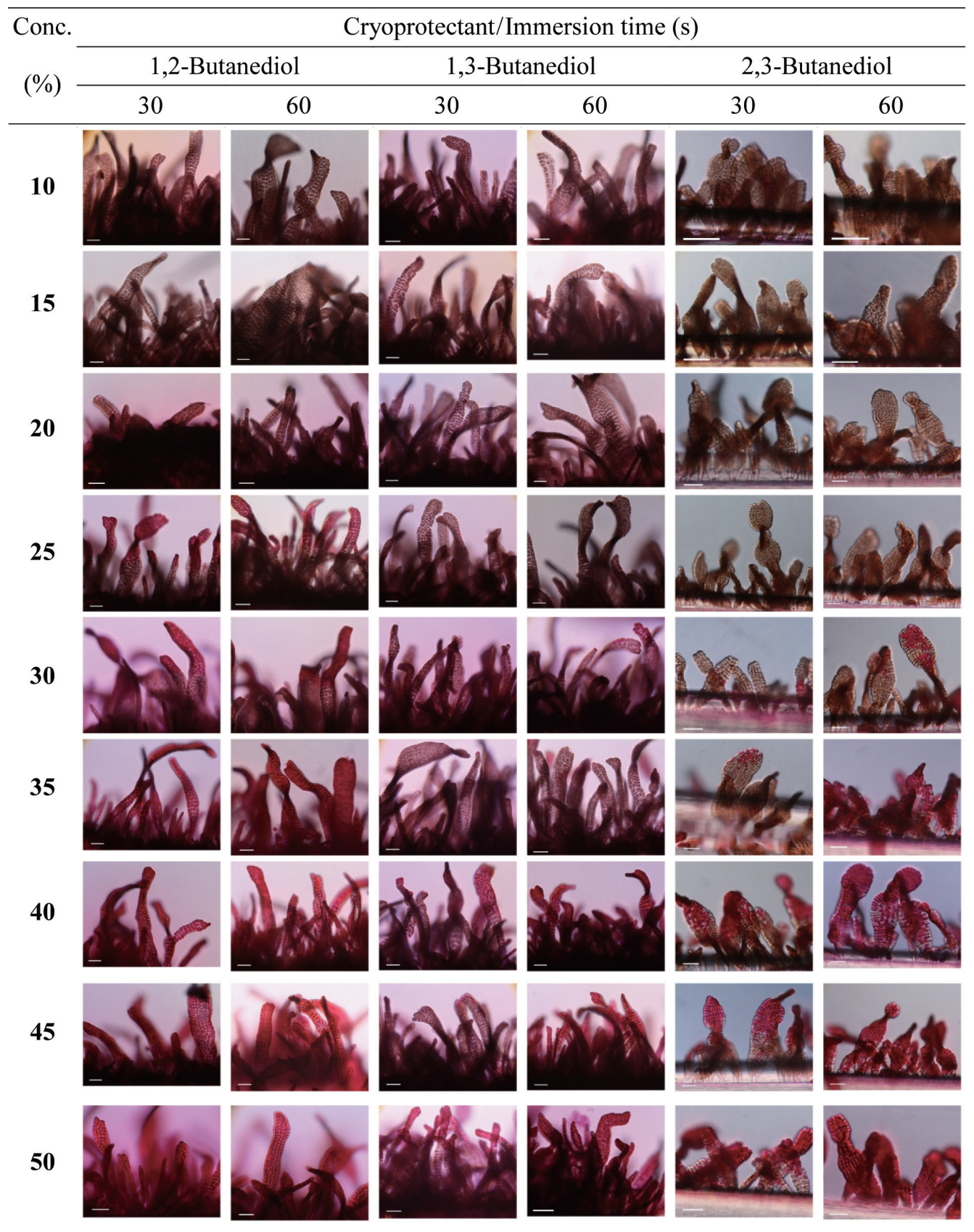

Fig. 1. Continued. 
Selection of a suitable CPA is important for successful cryopreservation. In general, DMSO, GC and methanol have been widely used in algae (Taylor and Fletcher, 1999). DMSO is the most-commonly used CPA for macroalgae, Porphyra spp. (Kuwano et al., 1996; Liu et al., 2004; Zhou et al., 2007), Gracilaria spp. (van der Meer and Simpson, 1984), and microalgae (Cañavate and Lubian, 1994). Glycerol is a polyol with three hydroxyl groups and is a highly hydroscopic, viscous, odorless, and sweet-tasting fluid of low toxicity (Fluhr et al., 2008). In this study, glycerol was less toxic than DMSO. Gwo et al. (2005) reported low glycerol toxicity in the microalgae, Nannochloropsis oculata at room temperature.

CPA toxicity depends on species, developmental stage and storage temperature. In particular, it is known that DMSO is less toxic at $0-5^{\circ} \mathrm{C}$ than at higher temperatures (Hubálek, 2003). Therefore, the viability of thalli may be enhanced by low temperature preservation.

CPA toxicity occurs after permeating into the cell, so the

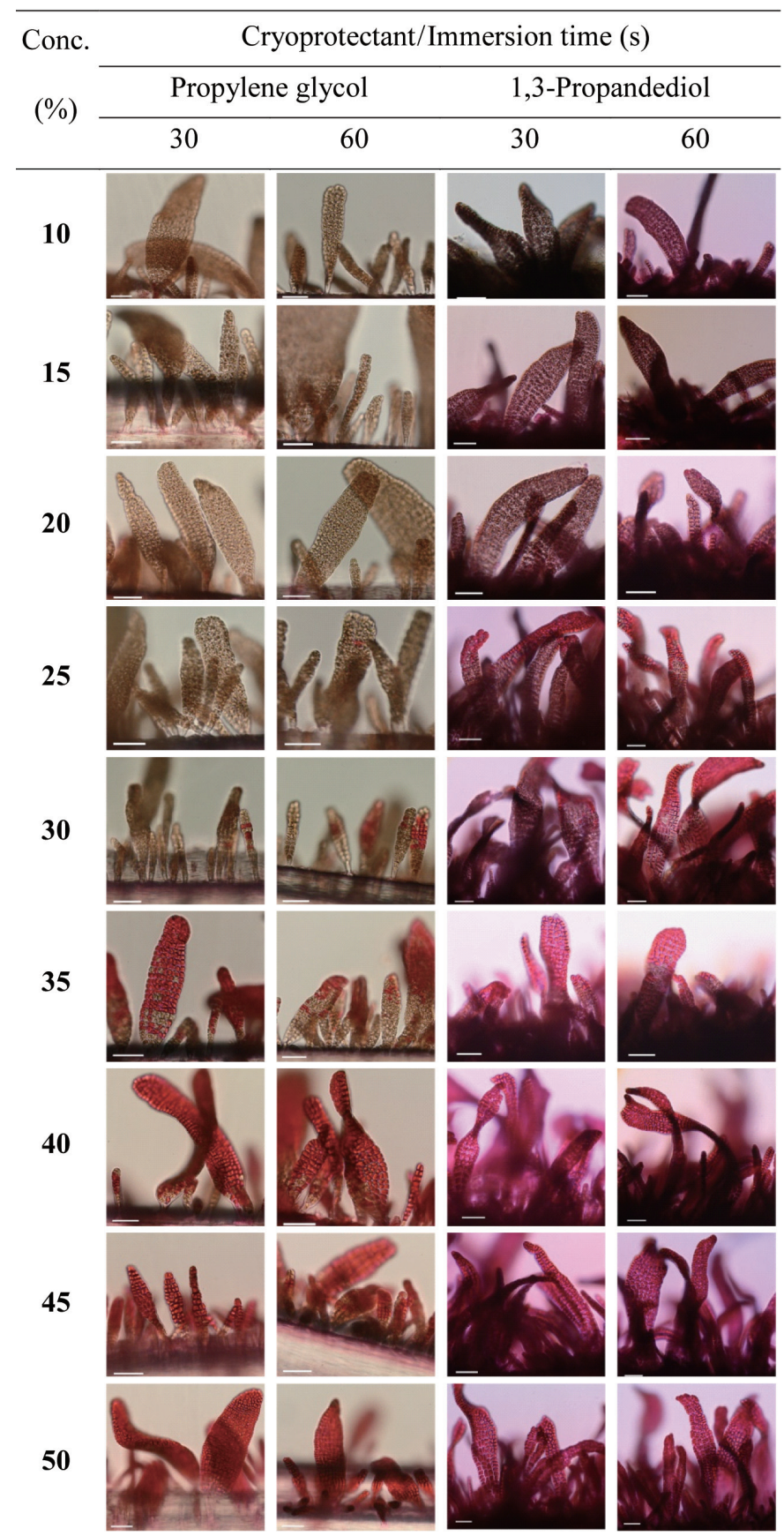

Fig. 1. Continued. 
more toxic CPAs are likely to be more membrane permeable, and vice versa. However, greater protection also requires more permeability (Zhang et al., 2005). To date, CPA concentrations before freezing have been considered because they are cryoprotective to cells, but induce cryoinjury in themselves (Fahy, 1986). Moreover, determining the optimum CPA requires a process of trial and error. Therefore, more investigation is required to determine the optimum CPA and its relationship with chemical reactions, temperature, and so on.

\section{References}

Baudot A, Peyridieu JF, Boutron P, Mazuer J and Odin J. 1996. Effect of saccharides on the glass-forming tendency and stability of solutions of 2,3-butanediol, 1,2-propanediol, or 1,3-butanediol in water, phosphate-buffered saline, Euro-Collins solution, or Saint Thomas cardioplegic solution. Cryobiology 33, 363-375.

Cañavate JP and Lubian LM. 1994. Tolerance of six marine microalgae to the cryoprotectants dimethyl sulfoxide and methanol. J Phycol 30, 559-565.

Fahy GM. 1986. The relevance of cryoprotectant "Toxicity" to cryobiology. Cryobiology 23, 1-13.

Fahy GM. 2010. Cryoprotectant toxicity neutralization. Cryobiology 60 (3 Suppl), S45-S53.

Fluhr JW, Darlenski R and Surber C. 2008. Glycerol and the skin: holistic approach to its origin and functions. Br J Dermatol 159, 23-34.

Gwo JC, Chiu JY, Chou CC and Cheng HY. 2005. Cryopreservation of a marine microalgae, Nannochloropsis oculata (Eustigmatophyceae). Cryobiology 50, 338-343.

Hubálek Z. 2003. Protectants used in cryopreservation of microorganisms. Cryobiology 46, 205-229.

Kuwano K, Aruga Y and Saga N. 1996. Cryopreservation of clonal gametophytic thalli of Porphyra (Rhodophyta). Plant Sci 116, 117-
124.

Liu H, Yu W, Dai J, Gong Q, Yang K and Lu X. 2004. Cryopreservation of protoplasts of the alga Prophyra yezoensis by vitirfication. Plant Sci 166, 97-102.

Liu XH, Zhang T and Rawson DM. 2001. Differential scanning calorimetry studies of intraembryonic freezing and cryoprotectant penetration in zebrafish (Danio rerio) embryo. J Exp Zool 290, 299-310.

Provasoli L. 1968. Media and prospects for the cultivation of marine algae. In: Cultures and Collections of Algae, Proceedings of U.S. Japan Conference. Watanabe A and Hattori A, eds. Japanese Society of Plant Physiology, Kyoto, JP, pp. 63-75.

Shaw JM, Ward C and Trounson AO. 1995. Survival of mouse blastocysts slow cooled in propanediol or ethylene glycol is influenced by the thawing procedure, sucrose and antifreeze proteins. Theriogenology 43, 1289-1300.

Taylor R and Fletcher RL. 1999. Cryopreservation of eukaryotic algae: a review of methodologies. J Appl Phycol 10, 481-501.

van der Meer JP and Simpson FJ. 1984. Cryopreservation of Gracilaria tikvahiae (Rhodophyta) and other macrophytic marine algae. Phycologia 23, 195-202.

Wowk B. 2010. Thermodynamic aspects of vitrification. Cryobiology $60,11-22$.

Yang H, Norris M, Winn R and Tiersch TR. 2010. Evaluation of cryoprotectant and cooling rate for sperm cryopreservation in the euryhaline fish medaka Oryzias latipes. Cryobiology 61, 211-219.

Zhang T and Rawson DM. 1993. Cryoprotectant toxicity and permeability studies on zebrafish (Brachydanio rerio) embryos. Cryobiology 30,640 .

Zhang YZ, Zhang SC, Liu XZ, Xu YJ, Hu JH, Xu YY, Li J and Chen SL. 2005. Toxicity and protective efficiency of cryoprotectants to flounder (Paralichthys olivaceus) embryos. Theriogenology 63, 763-773.

Zhou W, Li Y and Dai J. 2007. Study on cryopreservation of Porphyra yezoensis conchocelis. J Ocean Univ China 6, 299-302. 